\title{
Approximations for Solute Transport Through Porous Media With Flow Transverse to Layering
}

\author{
D. A. BARRY and J. C. PARKER \\ Soil and Environmental Sciences, Virginia Polytechnic Institute and State University, Blacksburg, \\ Virginia, 24061, U.S.A.
}

(Received: 4 April 1986; revised: 17 July 1986)

\begin{abstract}
Analytical approaches for the prediction of solute transport in layered porous media are investigated for the case of flow perpendicular to the direction of layering. One approach involves the use of averaging techniques to treat the profile as an equivalent homogeneous medium. The method is demonstrated on hypothetical and laboratory-measured data sets and a criterion for validity of the method is given. The second approach involves the use of time convolution to predict breakthrough curves for layered systems on the assumption that layer interactions have no significant effect on transport. Accuracy criteria are derived by comparing moments of the exact and approximate solutions and it is found that the convolution method has broader applicability than the equivalent single-layer analysis. An extension of the convolution method to include consideration of nonequilibrium transport due to the presence of mobile-immobile regions is presented and demonstrated by analysis of laboratory breakthrough data from a two-layer system exhibiting mobile-immobile regions.
\end{abstract}

Key words. Moments, equivalent single layer, convolution approximation, mobile-immobile model, solute dispersion.

\section{Introduction}

Mass transport in layered porous media occurs in many natural geologic situations for which accurate predictions of chemical fluxes are necessary, e.g., to quantify hazardous waste transport. Although phenomenologically based (Kreft, 1981b), the convection-dispersion model is commonly used in a predictive role to describe the transport and spreading of dissolved chemicals in layered and nonlayered hydrologic systems, as well as in problems arising in fields of chemical and petroleum engineering (Moranville et al., 1977).

In contrast with the extensive body of literature dealing with many aspects of the convection-dispersion model for single-layer systems, not much attention has been given to multilayer porous media, particularly in terms of purely analytical methods. In this paper we consider the analysis of chemical transport in systems with flow perpendicular to layers which are each homogeneous and well-defined. Fluid flow in the systems is considered to be uniform and steady with a known flux. We assume, for simplicity, that the systems are composed of two layers corresponding, for example, to a lagoon with a clay liner between the waste and the underlying profile. The methods used can be applied to more than two layers, albeit with increasing algebraic and computational complexity. 
A number of papers (e.g., Brigham, 1974; Kreft and Zuber, 1978; Parker and van Genuchten, 1984; van Genuchten and Parker, 1984) have shown the necessity of distinguishing between flux and resident concentration detection modes when using the convection-dispersion model. For example, sectioning a soil core and measuring concentration over the soil volume corresponds to resident concentration. In practice, it often occurs that breakthrough curves at a fixed position are of primary concern and these are more properly analyzed in terms of flux concentrations (Parker, 1984). Thus we will consider flux concentrations while noting that marked differences between model predictions of flux and resident concentrations will appear only when the dispersivity is relatively large, as demonstrated by Parker and van Genuchten (1984).

Previous analytical approaches to this problem, most notably Shamir and Harleman (1967), follow the transfer function approach used in linear system analysis, e.g., as applied to electrical networks (LePage, 1961). Shamir and Harleman (1967) make the assumption that, with respect to solute transport, each layer acts independently. This allows each layer to be treated, mathematically, as if it were semi-infinite. The semi-infinite solution for the first layer is used to give the upper boundary condition for the second layer, and so on. As deduced by Shamir and Harleman (1967), this assumption implies that the order of the layers has no effect on predicted breakthrough curves and, thus, lengths of the layers in the profile with the same dispersivity can be summed and treated as a single layer. Shamir and Harleman (1966), as well as Selim et al. (1977), have presented experimental results indicating that layer ordering has little effect on measured effluent concentrations. However, on physical grounds, there is no reason to assume, a priori, that solute transport is unaffected by the order of the layers. This point will be considered in more detail subsequently.

Other investigations utilizing the same assumption as Shamir and Harleman (1967) include: for nonreactive transport, Bruch (1970), Moranville et al. (1977), Ultman and Blatman (1977) and Pendse et al. (1978); and for transport involving radionuclide decay, Gureghian and Jansen (1985). The analyses reported by all these authors were predominantly analytical. The results show clearly that the assumption of noninteracting layers is a useful one, given that the error involved is acceptably small. As well as quantifying this error, we formalize the approach so that it can easily be applied to other transport scenarios, e.g., diffusion of solute from mobile to immobile regions.

In practice, it will often be convenient to treat the layered system as an equivalent single layer for purposes of making predictions of the system response to a certain input disturbance. In essence, the procedure is to cast the transport parameters for the simple single-layer convection-dispersion model in terms of the more complex model. As noted by Fried (1975), the "idea of replacing a complex problem by an elementary well-known equivalent problem is very natural and is the base of the philosophy of the scale change."

This approach has been followed by a number of authors to simplify the 
analysis of nonequilibrium transport associated with diffusional or chemical kinetics (Passioura, 1971; Baker, 1977; De Smedt and Wierenga, 1984; Valocchi, 1985; van Genuchten, 1985; Parker and Valocchi, 1986). Marle et al. (1967) considered an equivalent layer approach to stratified media for the case of flow parallel to the direction of layering. Here we follow the time moment analysis of Marle et al. (1967) and Valocchi (1985). Based on Aris (1958), this method consists of equating the moments calculated for the various transport models with the moments of the simple linear equilibrium model. Aris (1958) showed that moments can be calculated from solutions in the Laplace domain while noting that distributions can be conveniently and relatively accurately defined in terms of their first few moments. These properties have great practical use, especially when exact analytical solutions are not available, as is the case for solute transport in layered soils.

Our purpose is to analyze breakthrough curves from a medium consisting of two distinct layers. The time moment method will be used to define the parameters of the two-layer system in terms of an equivalent single-layer model and give conditions under which the latter model will accurately simulate layered system breakthrough curves. Additionally, the approach of Shamir and Harleman (1967) is examined, checked for accuracy and applied to a more complex transport model.

\section{Mathematical Model}

The mathematical model for solute transport perpendicular to the stratification is given by the system of equations

$$
R_{i} \frac{\partial c_{i}}{\partial t}=\mathbf{D}_{i} \frac{\partial^{2} c_{i}}{\partial x^{2}}-\mathbf{v}_{i} \frac{\partial c_{i}}{\partial x}, \quad i=1, \ldots, n .
$$

The $n$ equations given by (1) apply to $n$ homogeneous soil layers, with each layer referred to by the subscript $i$. In the following analyses we take $n=2 . R_{i}, c_{i}, \mathbf{D}_{i}$ and $\mathbf{v}_{i}$ are the retardation factor (Lindstrom et al., 1967), solute flux concentration, hydrodynamic dispersion coefficient and mean pore-water velocity, respectively, for each layer. Distances in each layer are referenced to a common origin. Assuming a constant volumetric flow rate, $q$, for the entire system and taking the water contents, $\theta_{i}$, to be constant within each layer, the mean pore-water velocity for each layer is

$$
\mathbf{v}_{i}=q / \theta_{i} .
$$

For convenience we rewrite (1) as

$$
\frac{\partial c_{i}}{\partial t}=D_{i} \frac{\partial^{2} c_{i}}{\partial x^{2}}-v_{i} \frac{\partial c_{i}}{\partial x}, \quad i=1, \ldots, n
$$

where the retarded mean pore-water velocity is defined as $v_{i}=v_{i} / R_{i}$ and the 
retarded coefficient of dispersion is $D_{i}=\mathbf{D}_{i} / R_{i}$. In a multilayered soil, the mean retarded pore-water velocity between the origin and location $x$ is

$$
\bar{v}=\frac{q x}{R_{1} \theta_{1} L_{1}+\cdots+R_{n} \theta_{n}\left(x-L_{n-1}\right)}
$$

where $L_{i}$ denotes the thickness of the $i$ th layer.

The flux boundary conditions to be applied in the solution of (3) are (Kreft, 1981b)

$$
\begin{aligned}
& c_{0}(t)=c_{1}\left(0^{+}, t\right), \\
& c_{i}\left(L_{i}^{-}, t\right)=c_{i+1}\left(L_{i}^{+}, t\right), \\
& v_{i} \frac{\partial c_{i}\left(L_{i}^{-}, t\right)}{\partial x}=v_{i+1} \frac{\partial c_{i+1}\left(L_{i}^{+}, t\right)}{\partial x}
\end{aligned}
$$

and

$$
\lim _{x \rightarrow \infty} c_{i}(x, t)=\text { finite, }
$$

where $c_{0}(t)$ is the concentration of the influent solution. Since we are considering a two-layer system, in the following we take the length of the first layer to be $L$, while the second layer is semi-infinite. We impose a uniform initial condition such that

$$
c_{i}=0, \quad x>0, t=0
$$

while noting that there is no loss of generality in imposing (9) since superposition may be used to construct solutions for arbitrary initial conditions from the solution subject to (9) (e.g., see van Genuchten and Alves, 1982).

The solution for the second layer in the Laplace domain, $\bar{c}_{2}(x, s)$, is (Kreft, 1981a)

$$
\bar{c}_{2}(x, s)=\frac{2 \bar{c}_{0}(s) q_{1}\left(1+q_{2}\right) \exp \left[v_{1} L\left(1-q_{1}\right) /\left(2 D_{1}\right)+v_{2}(x-L)\left(1-q_{2}\right) /\left(2 D_{2}\right)\right]}{\left(1+q_{1}\right)\left(q_{2}+q_{1}\right)-\left(1-q_{1}\right)\left(q_{2}-q_{1}\right) \exp \left[-q_{1} v_{1} L / D_{1}\right]}
$$

where $q_{i}=\left(1+4 s D_{i} / v_{i}^{2}\right)^{1 / 2}, s$ is the Laplace transform variable and $\bar{c}_{0}(s)$ is the Laplace transform of $c_{0}(t)$. Note that there is no difficulty in writing the solution for $\bar{c}_{1}(x, s)$ which has a form similar to (10). However, our interest is in breakthrough curves as affected by the layering and hence we consider only the second-layer flux concentration solution.

'Exact' solutions to (10) for $c_{2}(x, t)$ can be found by numerical inversion using readily available algorithms (e.g. Javendel et al., 1984). In common with any numerical solution, this method, which is undoubtedly useful in some applications, gives little understanding of the behavior of the system under consideration. For a profile consisting of many layers, a Laplace domain solution is 
not necessarily available. Furthermore, any numerical method may be subject to instability for certain combinations of the parameters, especially in the case of more complex systems. Later we consider a layered profile containing mobileimmobile regions for which numerical inversion gave inaccurate results. Thus, not only does the following analysis give insight into transport in layered profiles, the approximations may be more widely applicable and numerically stable than Laplace transform inversion.

\section{Analysis and Discussion}

\subsection{TIME-MOMENT ANALYSIS}

Aris (1958) has shown that a finite quantity of material moving in a dispersive medium possesses a set of time moments, $m_{j}$, defined by

$$
m_{j}=\int_{0}^{\infty} t^{j} c(x, t) \mathrm{d} t, \quad j=0,1,2, \ldots
$$

where $c(x, t)$ describes the concentration distribution in space and time. The moments given by (11) can be normalized with respect to $m_{0}$, the total mass injected per unit area. For example $m_{1} / m_{0}(\equiv \bar{\mu})$, is the normalized mean residence time which we refer to subsequently simply as the mean. Higher-order moments are commonly expressed relative to the mean by the central moments

$$
\mu_{j}=\frac{1}{m_{0}} \int_{0}^{\infty}[t-\bar{\mu}]^{i} c(x, t) \mathrm{d} t .
$$

Thus $\mu_{0}=1$ and $\mu_{1}=0$. The variance of the distribution is given by

$$
\mu_{2}=\frac{m_{2}}{m_{0}}-\vec{\mu}^{2}
$$

and the skewness is

$$
\mu_{3}=\frac{m_{3}}{m_{0}}-3 \bar{\mu} \mu_{2}-\bar{\mu}^{3}
$$

which is also expressed as the coefficient of skewness (Skopp, 1984)

$$
S=\mu_{3}\left(\mu_{2}\right)^{-3 / 2} \text {. }
$$

Observe that $c(x, t)$ need not be known explicitly to evaluate the moments since $m_{j}$ can be calculated from (Aris, 1958)

$$
m_{j}=(-1)^{j} \lim _{s \rightarrow 0}\left[\frac{\mathrm{d}^{j} \bar{c}(x, s)}{\mathrm{d} s^{j}}\right]
$$

which, in general, is a straightforward calculation. 


\subsection{AN EQUIVALENT SINGLE-LAYER}

Table I gives expressions for the mean, variance and skewness of the single- and two-layer system breakthrough curves obtained via (10) for a Dirac surface condition, i.e. (Kreft, 1981a)

$$
c_{0}(t)=\frac{m_{0} \delta(t)}{q}
$$

For the single-layer results, a subscript $e$ has been used to denote 'equivalent' two-layer parameters. Equating single- and two-layer means yields

$$
v_{e}=\frac{x}{\frac{L}{v_{1}}+\frac{x-L}{v_{2}}} .
$$

Note, as we expect, $v_{e}=\bar{v}$ in (4) when the latter equation is written for the two-layer case. Similarly, equating the variances in Table I gives an equation for the equivalent dispersion coefficient, $D_{e}$

$$
D_{e}=\frac{x^{2}}{\left[\frac{L}{v_{1}}+\frac{x-L}{v_{2}}\right]^{3}}\left\{\frac{D_{1} L}{v_{1}^{3}}+\frac{D_{2}(x-L)}{v_{2}^{3}}+\frac{D_{1}}{v_{1}^{2}}\left[\frac{D_{2}}{v_{2}^{2}}-\frac{D_{1}}{v_{1}^{2}}\right]\left[1-\exp \left(-P_{1}\right)\right)\right\}
$$

where $P_{1}=v_{1} L / D_{1}$. We note that $D_{e}$ and $v_{e}$ are functions of position. Although the entries in Table I were calculated for a Dirac (instantaneous) condition, the calculated equivalent single layer parameters are independent of the surface condition duration. However, differences between the equivalent single-layer and the exact two-layer breakthrough curves will decrease as the duration of the

Table I. One- and two-layer measures of central tendency calculated for a Dirac surface condition

\begin{tabular}{llll}
\hline $\bar{\mu}$ & One layer & Two layer (exact) & Two layer (convolution) \\
\hline $\bar{\mu}$ & $\frac{x}{v_{e}}$ & $\frac{L}{v_{1}}+\frac{x-L}{v_{2}}$ & $\frac{L}{v_{1}}+\frac{x-L}{v_{2}}$ \\
$\mu_{2}$ & $\frac{2 x D_{e}}{v_{e}^{3}}$ & $\frac{2 D_{1}}{v_{1}^{2}}\left\{\frac{L}{v_{1}}+\left[\frac{D_{2}}{v_{2}^{2}}-\frac{D_{1}}{v_{1}^{2}}\right]\left[1-\exp \left(\frac{-v_{1} L}{D_{1}}\right)\right]\right\}+\frac{2 D_{2}(x-L)}{v_{2}^{3}}$ & $\frac{2 L D_{1}}{v_{1}^{3}}+\frac{2 D_{2}(x-L)}{v_{2}^{3}}$ \\
$\mu_{3}$ & $\frac{12 x D_{e}^{2}}{v_{e}^{5}}$ & $\frac{12 D_{1}}{v_{1}^{2}}\left[\frac{D_{2}^{2}}{v_{2}^{4}}+\frac{D_{1} D_{2}}{v_{1}^{2} v_{2}^{2}}-\frac{2 D_{1}^{2}}{v_{1}^{4}}\right]\left[1-\exp \left(\frac{-v_{1} L}{D_{1}}\right)\right]+$ & $\frac{12 L D_{1}^{2}}{v_{1}^{5}}+\frac{12 D_{2}^{2}(x-L)}{v_{2}^{5}}$ \\
& & $+\frac{12 L D_{1}^{2}}{v_{1}^{5}}\left[1+\exp \left(\frac{-v_{1} L}{D_{1}}\right)\right]+\frac{12 D_{2}^{2}(x-L)}{v_{2}^{5}}-$ & \\
& $-\frac{12 L D_{1} D_{2}}{v_{1}^{3} v_{2}^{2}} \exp \left(\frac{-v_{1} L}{D_{1}}\right)$
\end{tabular}


surface condition increases as has been observed in other cases where a similar approach was used (Valocchi, 1985; Parker and Valocchi, 1986).

By definition, breakthrough curves calculated assuming an equivalent single layer will have the same mean and variance as the two-layer system. An error criterion for breakthrough curves calculated using this procedure can be derived by requiring the relative difference between the exact and equivalent normalized skewness coefficients, calculated using Table I and (15), to be small. Note that the $\mu_{i}$ of Table I are normalized to dimensionless time (Nauman and Buffham, 1983), $T=t \bar{v} / x$, by dividing by $(x / \bar{v})^{i}$. Alternatively, the following empirical condition is extremely simple and thus may be more useful in practice. The Péclet number of the equivalent single layer $\left(P_{e}=v_{e} x / D_{e}\right)$ will be equal to the sum of the layer Péclet numbers $\left[P_{1}=v_{1} L / D_{1}\right.$ and $\left.P_{2}=v_{2}(x-L) / D_{2}\right]$ for two layers with the same dispersivities, i.e., $D_{1} / v_{1}=D_{2} / v_{2}$. In this case the ratio of the equivalent layer Péclet number to the sum of the Péclet numbers of the individual layers is unity. In practice, the greater the difference of this ratio from unity, the less accurate the equivalent single layer approximation becomes. We find that the equivalent single layer approximation is reasonably accurate if

$$
\frac{P_{e}}{P_{1}+P_{2}}>\frac{1}{2} \text {. }
$$

Figures 1 and 2, which give breakthrough curves for continuous solute injection,

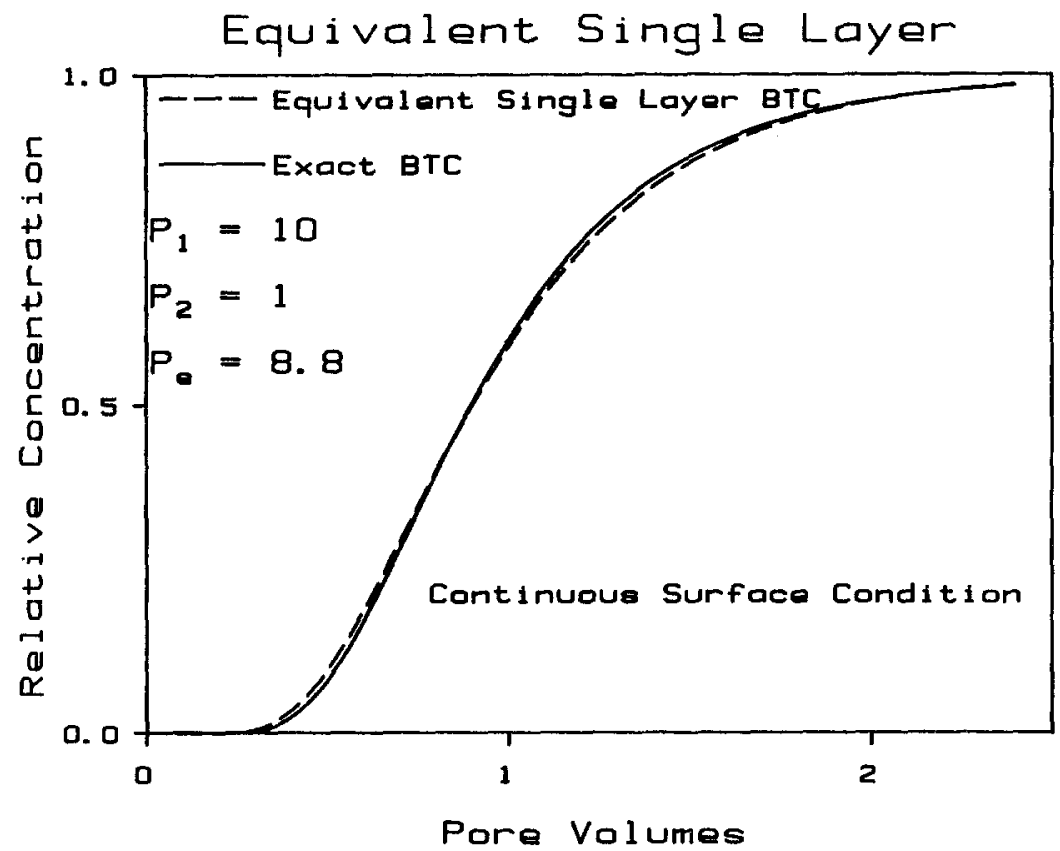

Fig. 1. Equivalent single-layer predictions when condition (19) is satisfied. Note that $x / L=1.5$. 


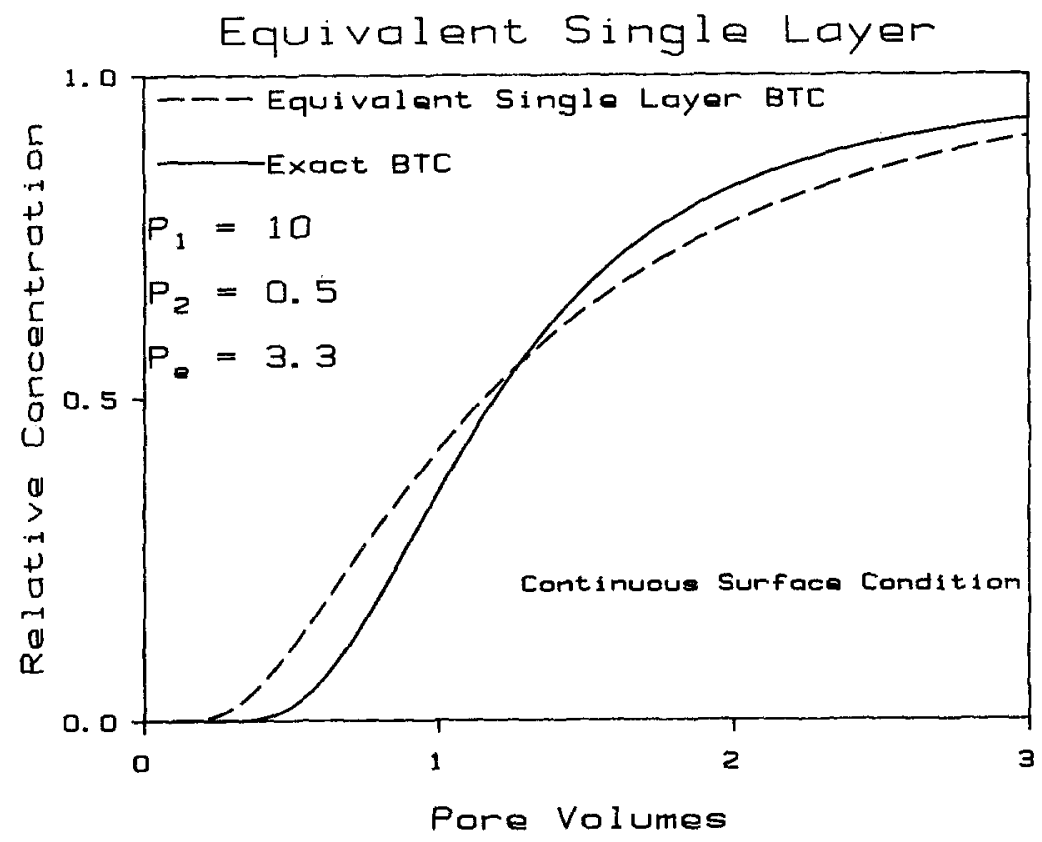

Fig. 2. Equivalent single-layer predictions when condition (19) is not satisfied. Note that $x / L=1.5$.

demonstrate the use of condition (19). In Figure 1, condition (19) is satisfied, while in Figure 2 it is not.

\subsection{EFFECT OF LAYER ORDERING}

The effect of switching the layer order in a profile may be quantified using the variance calculated for the two-layer system as given in Table I. Because the model assumes a finite first layer and a semi-infinite second layer, we expect that switching layers will generally result in different calculated variances. Note that the mean is unaffected by the layer order. It is of interest to determine when the layer ordering has a noticeable effect on breakthrough curves or, conversely, when it has little effect. Layer ordering may be presumed to have negligible effect on breakthrough curves if, for each ordering, the absolute difference between the variances is much less than the sum of the variances, i.e. if

$$
\frac{\frac{D_{2}}{v_{2}^{2}}\left[\frac{D_{2}}{v_{2}^{2}}-\frac{D_{1}}{v_{1}^{2}}\right]\left[1-\exp \left(-P_{2}\right)\right]}{\frac{D_{1} L}{v_{1}^{3}}+\frac{D_{2}(x-L)}{v_{2}^{3}}} \ll 1, \frac{D_{2} v_{1}^{2}}{D_{1} v_{2}^{2}}>1
$$


or,

$$
\frac{\frac{D_{1}}{v_{1}^{2}}\left[\frac{D_{1}}{v_{1}^{2}}-\frac{D_{2}}{v_{2}^{2}}\right]\left[1-\exp \left(-P_{1}\right)\right]}{\frac{D_{1} L}{v_{1}^{3}}+\frac{D_{2}(x-L)}{v_{2}^{3}}} \ll 1, \quad \frac{D_{2} v_{1}^{2}}{D_{1} v_{2}^{2}}<1
$$

hold. Qualitatively, we observe that condition (20) is satisfied if $D_{2} v_{1}^{2} /\left(D_{1} v_{2}^{2}\right) \simeq 1$, or if one layer is relatively long compared with the other.

\subsection{COMPARISON OF SEMI-INFINITE AND FINITE SOLUTIONS}

In laboratory tracer experiments, breakthrough curves are obtained from the soil-column effluent. Some uncertainty arises in the proper selection of the lower boundary condition in such experiments. When dispersion is predominantly hydrodynamic in nature and effluent is removed as it exits the column, experimental and theoretical analyses suggest a semi-infinite lower boundary may most adequately represent the system (Parker, 1984; Parker and Valocchi, 1986). However, when diffusion is the dominant component of the dispersion coefficient and the column is free-draining or an exit line exists in which dispersion is negligible, the boundary conditon to be applied at $x=L$, where $L$ is the column length, is $\partial c / \partial x=0$ (e.g., Wehner and Wilhelm, 1956). Even in this latter case, it is generally more convenient computationally to analyze effluent data using the solution for a semi-infinite system (Parlange and Starr, 1975).

If we interpret the boundary condition, $\partial c / \partial x=0$, to result from a vanishing dispersion coefficient beyond $x=L$, then the moments of Table I can be used to derive a condition for which the assumption of a semi-infinite system can be used without sacrificing accuracy. Putting $D_{2}=0$ and setting $x=L$ we again note from Table I that the semi-infinite and two-layer assumptions give identical means. By comparison of the normalized variances for each case we see that the semi-infinite solution will closely approximate breakthrough curve predictions obtained from the solution for a finite column if

$$
\left[1-\exp \left(-P_{1}\right)\right] / P_{1} \ll 1
$$

In agreement with Parlange and Starr (1978), we find that the assumption of a semi-infinite layer is justified for $P_{1}>16$. This comparison also predicts that the column Péclet number, $P_{1}$, can be obtained from the apparent Péclet number, $P_{\text {ap }}$, where the latter has been derived from breakthrough curve data analyzed using the solution for a semi-infinite column, via

$$
\frac{1}{P_{\text {ap }}}=\frac{1}{P_{1}}\left\{1-\frac{1}{P_{1}}\left[1-\exp \left(-P_{1}\right)\right]\right\} .
$$




\subsection{SEMI-INFINITE MEDIA APPROXIMATION: THE CONVOLUTION INTEGRAL}

If we approximate a 'spike' of solute having mass per unit area $m_{0}$ injected at the soil surface $(x=0)$, as a Dirac condition, then its distribution is given by

$$
c^{\delta}(x, t)=\frac{m_{0}}{\theta x} \bar{c}^{\delta}(x, t)
$$

where $\bar{c}^{\delta}$ is a transfer function representing the Dirac solution normalized to unit injected mass per pore volume. Convolution of (23) gives the solution for continuous injection as

$$
c(x, t)=\int_{0}^{t} \dot{m}_{0}(\tau) \bar{c}^{\delta}(x, t-\tau) /(\theta x) \mathrm{d} \tau .
$$

In (23), $m_{0}$ has been replaced by $\dot{m}_{0}(\tau) \mathrm{d} \tau$ where $\dot{m}_{0}$ is the mass flux density of material at $x=0$. For influent concentration $c_{0}(t)$ at hydraulic flux $q$ we have

$$
\dot{m}_{0}=q c_{0}
$$

and so, for $\mathbf{v} \equiv q / \theta$,

$$
\frac{\dot{m}_{0}}{\theta}=c_{0} \mathbf{v}
$$

Equation (24) then becomes

$$
c(x, t)=\frac{\mathbf{v}}{x} \int_{0}^{t} c_{0}(\tau) \bar{c}^{\delta}(x, t-\tau) \mathrm{d} \tau .
$$

Generalizing to $n$ soil layers gives

$$
c\left(x_{n}, t\right)=\frac{\mathbf{v}_{n}}{L_{n}} \int_{0}^{t} c\left(x_{n-1}, \tau\right) \bar{c}^{\delta}\left(L_{n}, t-\tau\right) \mathrm{d} \tau
$$

where $x_{i}$, for $i<n$, is the distance from the surface to the end of the $i$ th layer, $x_{n}$ is the distance to the measurement point, $L_{n}=x_{n}-x_{n-1}$, and $\mathbf{v}_{n}=q / \theta_{n}$. We will refer to (28) as the convolution approximation.

For $c_{0}(t)=1$, the solution for $c(x, t)$ in a semi-infinite homogeneous medium is well known (Lapidus and Amundson, 1952)

$$
c(x, t)=\frac{1}{2}\left\{\operatorname{erfc}\left[\frac{x-v t}{(4 D t)^{1 / 2}}\right]+\exp \left[\frac{v x}{D}\right] \operatorname{erfc}\left[\frac{x+v t}{(4 D t)^{1 / 2}}\right]\right\} .
$$

For this case $\bar{c}^{\delta}(x, t)$ is calculated by equating (27) and (29) and differentiating with respect to time,

$$
\bar{c}^{\delta}(x, t)=\frac{x^{2}}{v t(4 \pi D t)^{1 / 2}} \exp \left[-\frac{-(x-v t)^{2}}{4 D t}\right] .
$$

We wish to investigate when the convolution approximation will accurately 
predict breakthrough curves from two soil layers. To do this using the moment method, we observe that the Laplace solution corresponding to (28) for two layers with integrand defined by (29) and (30) is equal to the exponential term in the numerator of (10) multiplied by $\bar{c}_{0}(s)$. Note that this approximation of (10) may be obtained by taking a first-order Taylor series expansion of the nonexponential terms in (10) which will be accurate when $s D_{i} / v_{1}^{2} \ll 1$. Again we assume a Dirac condition and calculate moments for this approximation from the Laplace domain solution. These results have been included in Table I. The convolution approximation has the same mean as the exact two-layer mean. The approximation will yield accurate predictions if there is little difference between the exact and approximate variances, or

$$
\frac{\left|\frac{D_{2}}{v_{2}^{2}}-\frac{D_{1}}{v_{1}^{2}}\right| \frac{D_{1}}{v_{1}^{2}}\left[1-\exp \left(-P_{1}\right)\right]}{\frac{D_{1} L}{v_{1}^{3}}+\frac{D_{2}(x-L)}{v_{2}^{3}}} \ll 1
$$

Although not identical, condition (31) and condition (20) are very similar and will hold under the same general physical criteria. In the majority of cases then, where it is found that the layer ordering has little effect on the breakthrough curve, we expect that the convolution approximation will be accurate (and vice versa).

To obtain the transfer function for the equilibrium convection-dispersion equation given by (30) we equated (27) and (29) and differentiated with respect to time as noted above. This method can be applied easily to any single-layer model. For example, consider the nonequilibrium convection-dispersion model for a soil with mobile-immobile zones (De Smedt and Wierenga, 1979)

$$
R_{m} \theta_{m} \frac{\partial c_{m}}{\partial t}+R_{i m} \theta_{i m} \frac{\partial c_{i m}}{\partial t}=\theta_{m} \mathbf{D}_{m} \frac{\partial^{2} c_{m}}{\partial x^{2}}-q \frac{\partial c_{m}}{\partial x}
$$

and

$$
R_{i m} \theta_{i m} \frac{\partial c_{i m}}{\partial t}=\alpha\left(c_{m}-c_{i m}\right)
$$

where the subscripts $m$ and $i m$ refer to the mobile and immobile zones, respectively, and $\alpha$ is the mass transfer coefficient. For a continuous injection of solution applied at $x=0$, the solution to (32) and (33) is

$$
c_{m}(x, t)=\frac{\mathbf{v}}{x} \int_{0}^{t} c(x, \tau) J\left[\frac{\alpha \tau}{R_{m} \theta_{m}}, \frac{\alpha(t-\tau)}{R_{i m} \theta_{i m}}\right] \mathrm{d} \tau
$$

where $c(x, \tau)$ is given by (30) written for the mobile region parameters, $D=$ $D_{m} / R_{m}$ and $v=v_{m}=q /\left(\theta_{m} R_{m}\right)$, and $J(a, b)$ is the Goldstein $J$-function (Gold- 
stein, 1953):

$$
J(a, b)=1-\exp (-b) \int_{0}^{a} \exp (-\tau) I_{0}\left[2(b \tau)^{1 / 2}\right] \mathrm{d} \tau,
$$

where $I_{i}$ denotes an $i$ th order modified Bessel function. For this case the transfer function is

$$
\begin{aligned}
c_{m}^{\delta}(x, t)= & \frac{\alpha}{\left(R_{m} \theta_{m} R_{i m} \theta_{i m}\right)^{1 / 2}} \int_{0}^{t} c(x, \tau) \exp \left[-\frac{\alpha \tau}{R_{m} \theta_{m}}-\frac{\alpha(t-\tau)}{R_{i m} \theta_{i m}}\right]\left[\frac{\tau}{t-\tau}\right]^{1 / 2} \times \\
& \times I_{1}\left\{2 \alpha\left[\frac{\tau(t-\tau)}{R_{m} \theta_{m} R_{i m} \theta_{i m}}\right]^{1 / 2}\right\} \mathrm{d} \tau+\exp \left[-\frac{\alpha t}{R_{m} \theta_{m}}\right] c(x, t) .
\end{aligned}
$$

When computing the integral on the right-hand side of (36), it is useful to note that as $\tau \rightarrow 0$ and $t$, the integrand $\rightarrow 0$ and

$$
c(x, t) \exp \left[-\alpha t /\left(R_{m} \theta_{m}\right)\right] \alpha t /\left(R_{m} \theta_{m} R_{i m} \theta_{i m}\right)^{1 / 2},
$$

respectively.

\section{Application to Experimental Data}

\subsection{SHAMIR AND HARLEMAN (1967)}

We now apply the above methods to data from experiments reported by Shamir and Harleman (1967) involving continuous injection of $\mathrm{NaCl}$ into a medium consisting of several alternating $3.175 \mathrm{~cm}$ thick layers of two different granular silica sands. The condition of no retardation is assumed. Shamir and Harleman (1967) give the dispersion coefficients for the two materials as $0.0115 \mathrm{~cm}^{2} \mathrm{~h}^{-1}$ and $0.0465 \mathrm{~cm}^{2} \mathrm{~h}^{-1}$, for a pore water velocity of $0.154 \mathrm{~cm} \mathrm{~h}^{-1}$. We note that for these parameters, conditions (19), (20) and (31) are all satisfied. Because layer ordering does not affect the breakthrough curve, the lengths of the alternating layers can be summed, and the system analyzed as if it consisted of two layers with lengths of, for layer $1,41.6 \mathrm{~cm}$ and, for layer $2,41.3 \mathrm{~cm}$. The results of the convolution approximation, via (28), with $c\left(x_{n-1}, t\right)$ given by (29), and the transfer function by (30), previously given by Shamir and Harleman (1967), is shown as the solid line in Figure 3. This prediction was checked by comparison with a numerical solution which yielded an identical breakthrough curve.

For a finite-duration input signal, it is of interest to note that although the equivalent single layer has the same mean and variance as the exact two-layer model, its predictions are less accurate than the convolution approximation (Figure 3), which has the same mean but different variance. (This statement is meaningless in the case of a continuous input although, as already noted, the equivalent layer parameters remain unaffected.) The reason for this is that the calculated moments of second order and higher can be affected noticeably by the 
Experimental Data and Predictions

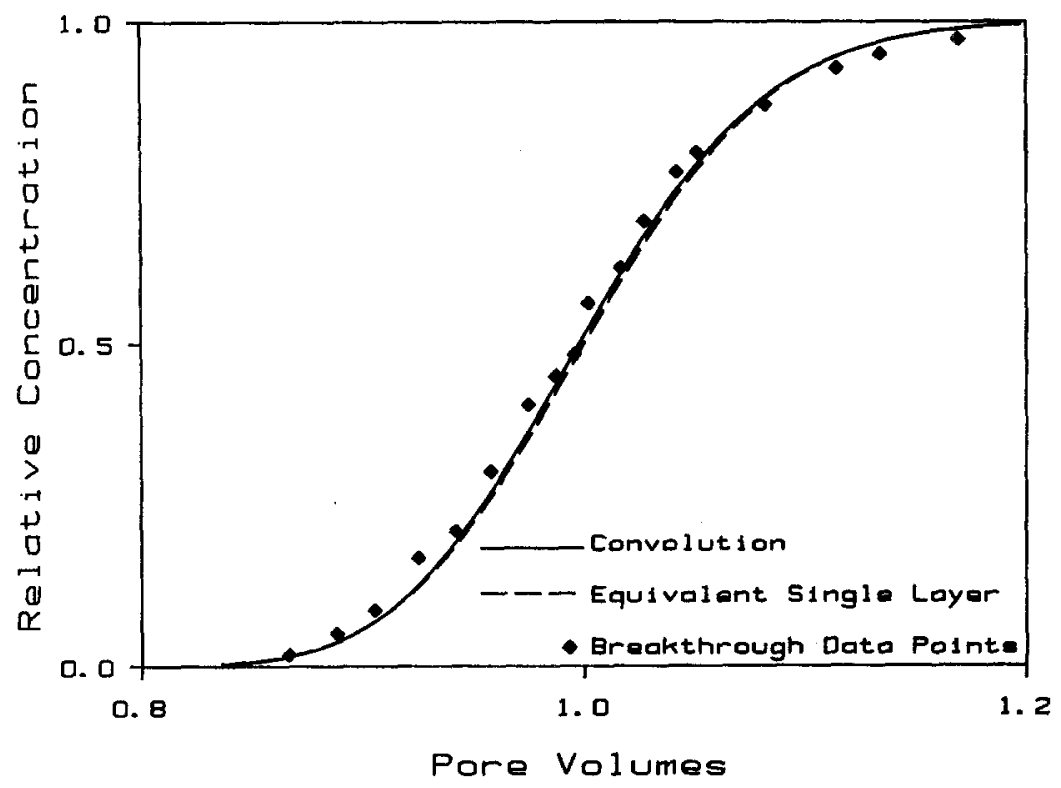

Fig. 3. Approximations compared with experimental data from Shamir and Harleman (1967).

tail of the distribution, in which case the distribution's skewness should be considered explicitly in the calculation of the equivalent parameters. An alternative approach is to calculate the equivalent parameters using weighted moments, which weight the main body of the signal at the expense of the less-important tail. The most useful weighting function is $\exp (-\chi t)$, where $\chi$ is the weighting factor. Equation (11) becomes

$$
w_{j}=\int_{0}^{\infty} t^{j} \exp (-\chi t) c(x, t) \mathrm{d} t, \quad j=0,1,2, \ldots
$$

where the $w_{j}$ are the exponentially weighted moments. Unweighted moments given by (11) are calculated using $\chi=0$. Nauman and Buffham (1983) suggest the mean as a reasonable weighting factor.

\subsection{PANIGATTI (1970)}

Panigatti (1970) measured $\mathrm{Cl}$ breakthrough curves from homogeneous and two-layered soil columns. Evidence of mobile-immobile soil regions was observed. The breakthrough curves used here are for Norge loam, from Perkins, Oklahoma, and Stratford I soil, from Stratford, Oklahoma. The porosities of these soils were 0.345 and 0.243 , respectively, and the experimental hydraulic flux was fixed at $1.78 \mathrm{~cm} \mathrm{~h}^{-1}$. The columns in the study were $30 \mathrm{~cm}$ long (layers 
Stratford I

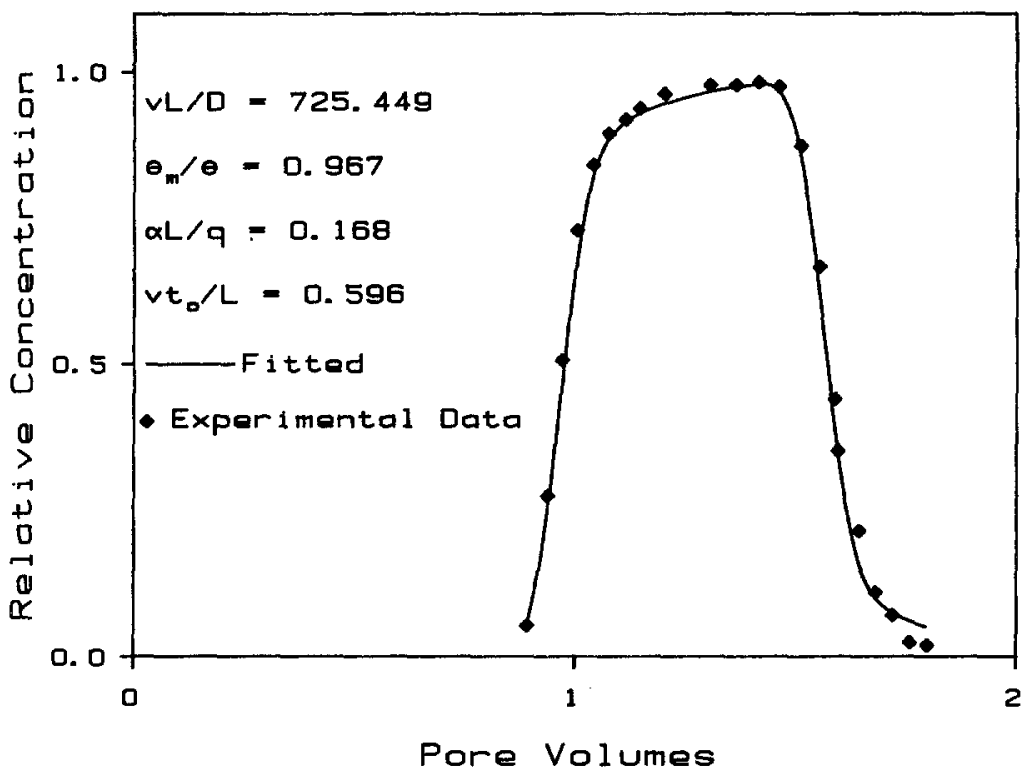

Fig. 4. Best fit of the mobile-immobile model to experimental data from Panigatti (1970) for the Stratford I soil.

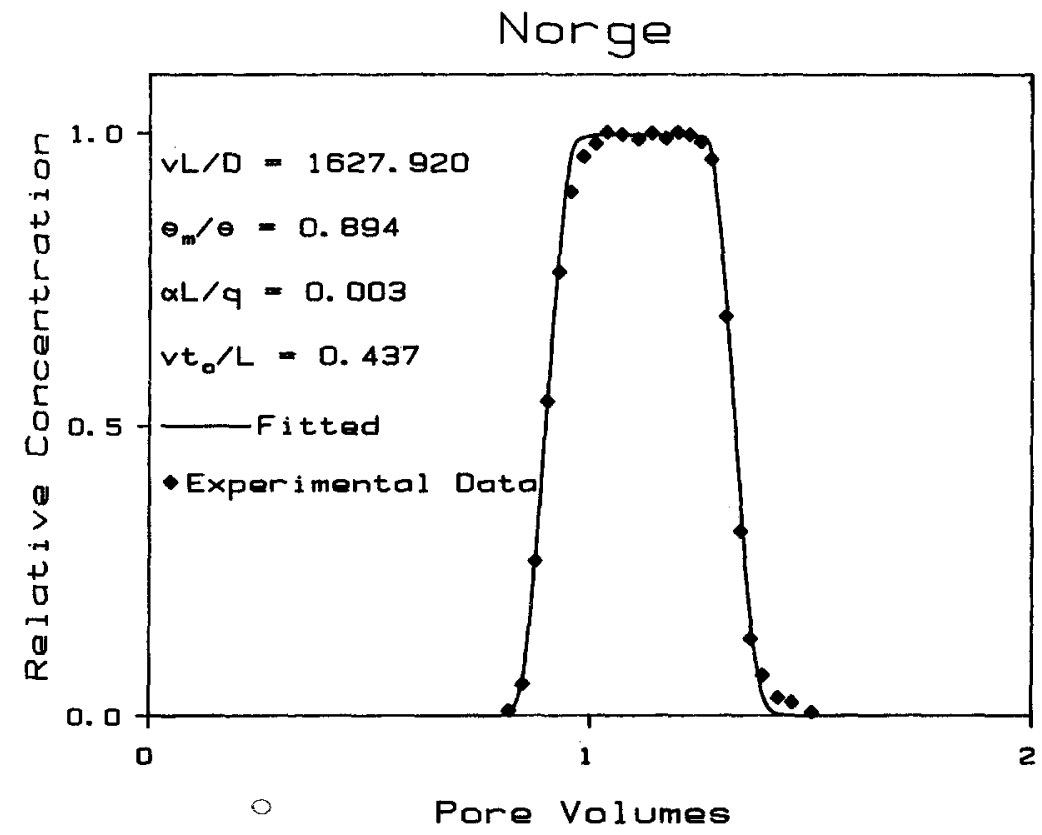

Fig. 5. Best fit of the mobile-immobile model to experimental data from Panigatti (1970) for the Norge soil. 
of $15 \mathrm{~cm}$ were used for the layered experiments) with a diameter of $7.5 \mathrm{~cm}$. Solute was applied as a pulse in each experiment. We found that the mass of $\mathrm{Cl}$ calculated by integrating under the given breakthrough curves differed slightly from the input quantities given by Panigatti (1970). We assume that no irreversible reactions of $\mathrm{Cl}$ take place and so the durations of the applied solute pulses were adjusted to conserve mass of $\mathrm{Cl}$.

Figures 4 and 5 show the measured and fitted breakthrough curves for the Stratford I and Norge soils, respectively, obtained using the parameters listed in each figure assuming retardation factors of unity. In this case we make use of the convolution approximation, substituting (34) and (36) into (28). To ascertain the numerical precision of the convolution calculation, results for the homogeneous Stratford I soil, treated computationally as two identical $15 \mathrm{~cm}$ layers, were checked against the exact, single-layer solution given by (34). No discernible difference between the one- and two-layer curves was observed.

Panigatti (1970) found no significant difference between breakthrough curves for the Norge-Stratford I layering or the reverse order. In Figure 6 we show the approximation's accurate predictions for the: Norge-Stratford I ordering.

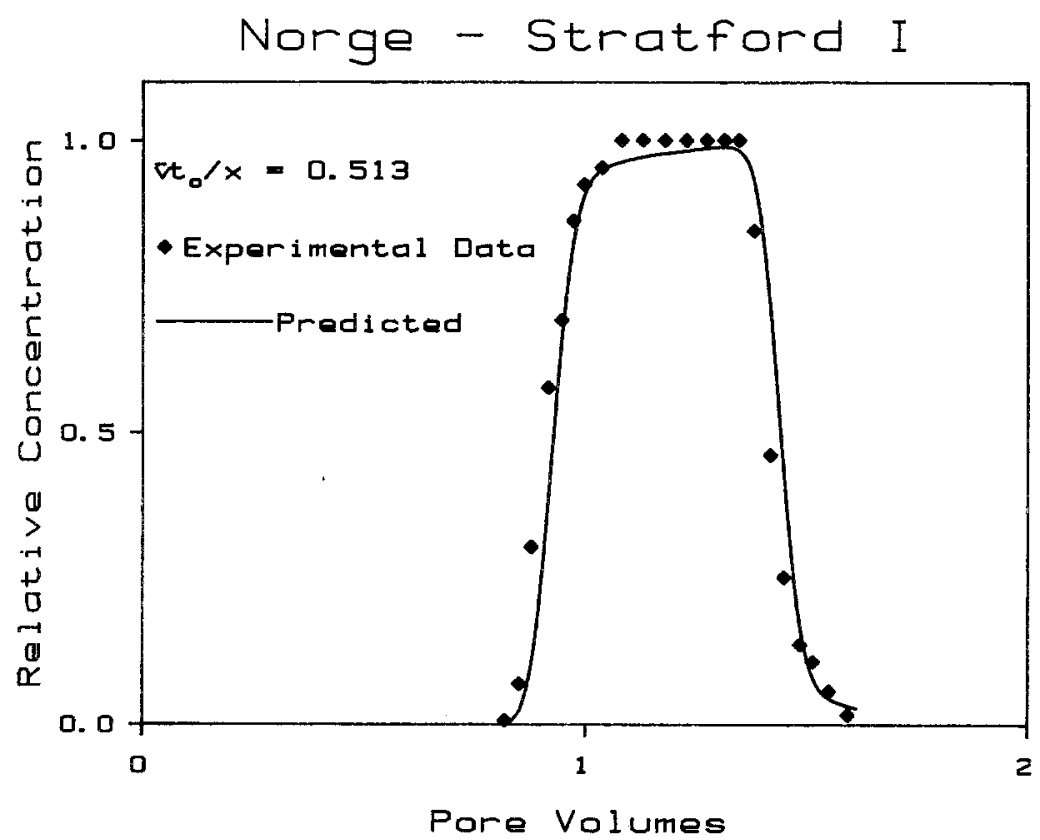

Fig. 6. Convolution approximation prediction of the breakthrough curve compared with experimental data from Panigatti (1970) for two $15 \mathrm{~cm}$ layers consisting of Norge overlaying Stratford I. 


\section{Conclusions}

Analytical approaches to transport in layered media may employ averaging techniques to treat the profile as a homogeneous single layer, or treat each layer independently as if there were no interactions between layers and make use of the convolution integral. We have analyzed the feasibility and validity of these approximations with particular reference to predictions of breakthrough curves from two layers. The results of this study can be very useful in practice. For example, Monte Carlo simulations of field solute transport generally require large numbers of realizations of the random process to be generated, and hence substantial computer time, for reasonable estimates of population means and variances (e.g., Persaud et al., 1985). This problem is compounded for layered systems. Thus, if accuracy criteria are satisfied, analytical approximations such as those given here can markedly reduce computational costs without loss of precision in the results.

It is apparent that the simplest and most straightforward way to predict breakthrough curves from a two-layer system is to use the equivalent single-layer approximation. Transport parameters for the equivalent layer are easily found by equating means and variances for the one- and two-layer systems.

A more accurate approximation is given by the convolution integral which assumes that layer interactions have no significant effect on solute transport. Again by comparing the moments of the approximation with the exact moments, a criterion for the validity of this approach was obtained. The approximation was also applied to experimental data using a transport model incorporating the assumption of mobile-immobile zones within the soil.

\section{Acknowledgements}

The authors acknowledge and express appreciation for support provided by the United States Environmental Protection Agency under grant number R-810819$01-0$.

\section{References}

Aris, R., 1958, On the dispersion of kinematic waves, Proc. Royal Soc. London A245, 268-277.

Baker, L. E., 1977, Effects of dispersion and dead-end pore volume in miscible flooding, Soc. Pet. Eng. J. 17, 219-227.

Brigham, W. E., 1974, Mixing equations in short laboratory cores, Soc. Pet. Eng. J. 17, 91-99.

Bruch, J. C., 1970, Two-dimensional dispersion experiments in a porous medium, Water Resour. Res. 6, 791-800.

De Smedt, F. and Wierenga, P. J., 1979, A generalized solution for solute flow in soils with mobile-immobile water, Water Resour. Res. 15, 1137-1141.

De Smedt, F. and Wierenga, P. J., 1984, Solute transfer through columns of glass beads, Water Resour. Res. 20, 25-232.

Fried, J. J., 1975, Groundwater Pollution, Elsevier, Amsterdam. 
Goldstein, F. R. S., 1953, On the mathematics of exchange processes in fixed columns. I. Mathematical solutions and asymptotic expansions, Proc. Roy. Soc. London 219, 151-185.

Gureghian, A. B. and Jansen, G., 1985, One-dimensional analytical solutions for the migration of a three-member radionuclide decay chain in a multilayered geologic medium, Water Resour. Res. 21, 733-742.

Javandel, I., Doughty, C. and Tsang, C. F., 1984, Groundwater Transport: Handbook of Mathematical Models, American Geophysical Union, Washington, D.C.

Kreft, A., 1981a, On the residence time distribution in systems with axial dispersed flow, Bull. Ac. Pol.: Tech. 29, 509-520.

Kreft, A., 1981b, On the boundary conditions of flow through porous media and conversion of chemical flow reactors, Bull. Ac. Pol.: Tech. 29, 521-529.

Kreft, A. and Zuber, A., 1978, On the physical meaning of the dispersion equation and its solution for different initial and boundary conditions, Chem. Eng. Sci. 33, 1471-1480.

Lapidus, L. and Amundson, N. R., 1952, Mathematics of adsorption in beds. VI. The effects of longitudinal diff usion in ion exchange and chromatographic columns. J. Phys. Chem. 56, 984-988.

LePage, W. R., 1961, Complex Variables and the Laplace Transform for Engineers, McGraw-Hill, New York.

Lindstrom, F. T., Haque, R., Freed, V. H., and Boersma, L., 1967, Theory on movement of some herbicides in soils. Linear diffusion and convection of chemicals in soil, Environ. Sci. Technol. 2, 561-565.

Marle, C., Simandoux, P., Pacsirszky, J., and Gaulier, C., 1967, Etude du déplacement de fluides miscibles en milieu poreux stratifié, Rev. Inst. Fr. Petrole. 22, 272-294.

Moranville, M. B., Kessler, D. P. and Greenkorn, R. A., 1977, Dispersion in layered porous media, A.I.Ch.E.J. 23, 786-794.

Nauman, E. B. and Buffham, B. A., 1983, Mixing in Continuous Flow Systems, John Wiley, New York.

Panigatti, J. L., 1970, The influence of soil textural stratification on chloride and fluometuron movement, unpublished MS Thesis, Oklahoma State University, Stillwater, Oklahoma.

Parker, J. C., 1984, Analysis of solute transport in column tracer studies, Soil Sci. Soc. Am. J. 48, 719-724.

Parker, J. C. and Valocchi, A. J., 1986, Constraints on the validity of equilibrium and first-order kinetic transport models in structured soils, Water Resour. Res. 22, 399-407.

Parker, J. C. and van Genuchten, M. Th., 1984, Flux-averaged and volume-averaged concentrations in continuum approaches to solute transport, Water Resour. Res. 20, 866-872.

Parlange, J.-Y. and Starr, J. L., 1975, Linear dispersion in finite columns, Soil Sci. Soc. Am. Proc. 39, 817-819.

Parlange, J.-Y. and Starr, J. L., 1978, Dispersion in soil columns: effect of boundary conditions and irreversible reactions, Soil Sci. Soc. Am. J. 42, 15-18.

Passioura, J. B., 1971, Hydrodynamic dispersion in aggregated media. 1. Theory, Soil Sci. 111, 339-344.

Pendse, H., Tien, C., Rajagopalan, R., and Turian, R. M., 1978, Dispersion measurement in clogged flter beds: a diagonistic study on the morphology of particle deposits, A.I.Ch.E.J. 24, 473-484.

Persaud, N., Giraldez, J. V., and Chang, A. C., 1985, Monte-Carlo simulation noninteracting solute transport in a spatially heterogeneous soil, Soil Sci. Soc. Am. J. 49, 562-568.

Selim, H. M., Davidson, J. M., and Rao, P. S. C., 1977, Transport of reaction solutes through multilayered soils, Soil Sci. Soc. Am. J. 41, 3-10.

Shamir, U. Y. and Harleman, D. R. F., 1966, Numerical and Analytical Solutions of Dispersion Problems in Homogeneous and Layered Aquifers, Tech. Rep. No. 89, Hydrodynamics Lab., Mass Inst. Technol., Cambridge, Mass., pp. 206.

Shamir, U. Y. and Harleman, D. R. F., 1967, Dispersion in layered porous media, Proc. Am. Soc. Civil Eng. Hydr. Div. 93, 236-260.

Skopp, J., 1984, Analysis of solute movement in structured soils, in J. Bouma and P. A. C. Raats (eds.), Proc. ISSS Symposium on Water and Solute Movement in Heavy Clay Soils, International Institute for Land Reclamation and Improvement, III, Wageningen: (IRLI pub. 37), pp. 220-227.

Ultman, J. S. and Blatman, H. S., 1977, A compartmental dispersion model for the analysis of mixing in tube networks, A.I.Ch.E.J. 23, 169-176. 
Valocchi, A. J., 1985, Validity of the local equilibrium assumption for modeling sorbing solute transport through homogeneous soils, Water Resour. Res. 21, 808-820.

van Genuchten, M. Th., 1985, A general approach for modeling solute transport in structured soil, Proc. 17th Int. Congress, Int. Assoc. of Hydrogeologists, Hydrogeology of Rocks of Low Permeability, Tucson, Arizona, January, 1985, Memoirs IAH 17, part 2, pp. 513-536.

van Genuchten, M. Th. and Alves, W. J., 1982, Analytical Solutions of the One-Dimensional Convection-Dispersion Equation, U.S. Department of Agriculture, Technical Bulletin No. 1661. $151 \mathrm{p}$.

van Genuchten, M. Th. and Parker, J. C., 1984, Boundary conditions for displacement experiments through short laboratory soil columns, Soil Sci. Soc. Am. J. 48, 703-708.

Wehner, J. F. and Wilhelm, R. H., 1956, Boundary conditions of flow reactor, Chem. Eng. Sci. 6, 89-93. 